\begin{tabular}{|c|l|}
\hline Title & Effect of carbon incorporation on electrical properties of n-type GaN surfaces \\
\hline Author(s) & Kimura, Takeshi; Hashizume, Tamotsu \\
\hline Citation & $\begin{array}{l}\text { Journal of A pplied Physics, 105(1), 014503 } \\
\text { https://doi.org/10.1063/_.3056395 }\end{array}$ \\
\hline Issue Date & 2009-01 \\
\hline Doc URL & http://hdl.handle.net/2115/35595 \\
\hline Rights & $\begin{array}{l}\text { Copyright 2009 A merican Institute of Physics. This article may be downloaded for personal use only. A ny other use } \\
\text { requires prior permission of the author and the A merican Institute of Physics. }\end{array}$ \\
\hline Type & article \\
\hline File Information & JA pplPhys_105_014503.pdf \\
\hline
\end{tabular}

Instructions for use 


\title{
Effect of carbon incorporation on electrical properties of $n$-type GaN surfaces
}

\author{
Takeshi Kimura ${ }^{\text {a) }}$ and Tamotsu Hashizume ${ }^{\text {b) }}$ \\ Research Center for Integrated Quantum Electronics (RCIQE) and Graduate School of Information Science \\ and Technology, Hokkaido University, North-13 West-8, Kita-ku, Sapporo 060-8628, Japan
}

(Received 15 October 2008; accepted 18 November 2008; published online 6 January 2009)

\begin{abstract}
We intentionally incorporated carbon into $n$-GaN by high-temperature annealing of a $\mathrm{SiN}_{x} / \mathrm{CN}_{x} / \mathrm{GaN}$ structure to study the effect of unintentional carbon incorporation on the electrical properties of $n$-type GaN surfaces. X-ray photoelectron spectroscopy results showed outdiffusion of $\mathrm{Ga}$ atoms from the GaN surface during high-temperature annealing even when the $\mathrm{SiN}_{x}$ layer was present. The current-voltage characteristics showed a drastic increase in current in the forward and reverse directions of the Schottky diode in the carbon-incorporated sample. They also showed no temperature dependence from 150 to $300 \mathrm{~K}$. The current-voltage curves of the carbon-incorporated samples in the forward and reverse directions could be almost completely reproduced by assuming an exponentially decaying distribution from the surface for shallow donors. (C) 2009 American Institute of Physics. [DOI: 10.1063/1.3056395]
\end{abstract}

\section{INTRODUCTION}

Carbon and oxygen are impurities that may contaminate GaN-based devices when they are being fabricated. For example, the ion implantation technique is one of the most commonly used processes in semiconductor technology because it can introduce a well-defined impurity doping profile in the selected region. For GaN-based devices, however, high-temperature annealing $\left(1000-1300{ }^{\circ} \mathrm{C}\right)$ is necessary for effective activation of impurities in the donor or acceptor state and for recovery from implantation-induced crystalline defects. ${ }^{1-3}$ Even if one utilizes a protection layer on the GaN surface, high-temperature annealing processes will cause electrical degradation of the surface associated with the decomposition (such as out diffusion of $\mathrm{Ga}$ and/or $\mathrm{N}$ atom) and/or unintentional impurity (such as carbon and oxygen) incorporation.

In particular, carbon is known as amphoteric impurity in III-V semiconductors. In fact, the amphoteric behavior of carbon was reported for GaAs, AlAs, and InAs grown by molecular beam epitaxy (MBE). ${ }^{4}$ On the analogy of $p$-type doping of GaAs with $\mathrm{C}$, several works were devoted to dope carbon into GaN. Hirota et al. ${ }^{5}$ reported that the binding energy of the substitutional $\mathrm{C}$ at the $\mathrm{N}$ site $\left(\mathrm{C}_{\mathrm{N}}\right)$ was 203 $\mathrm{meV}$ in the carbon-doped $\mathrm{GaN}$ by an ion implantation or a gas phase diffusion using $\mathrm{C}_{2} \mathrm{H}_{6}$. However, the $p$-type conduction was not observed in both samples. Efforts to produce $p$-type $\mathrm{GaN}$ : $\mathrm{C}$ have had only limited success. The $\mathrm{C}$ doping during epitaxial growth of $\mathrm{GaN}$ typically produces a highly resistive layer, ${ }^{6}$ except for the $p$-type conductivity in cubicphase GaN grown on GaAs by rf-assisted MBE using electron-beam evaporation of graphite. ${ }^{7}$ The difficulty of the $p$-type conduction by carbon in a wurtzite structure may be attributed to the fact that the formation energy of the $C_{N}$ is not small on the surface of $p$-type material. ${ }^{8}$ Moreover, on

\footnotetext{
a)Electronic mail: kimura@rciqe.hokudai.ac.jp.

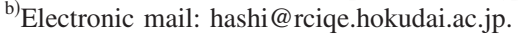

the Ga-terminated (0001) surface, the substitutional doping of carbon on the $\mathrm{N}$ site may not be efficient. ${ }^{9}$ Thus, the amphoteric nature on the carbon-related levels in GaN seems to depend on the crystalline quality of $\mathrm{GaN}$ and the device processing condition. However, no study that we know of has reported on the influence of unintentional incorporation of carbon impurities into $\mathrm{GaN}$ during high-temperature annealing.

The purpose of this paper is to investigate the effect of carbon incorporation on the electrical properties of $n$-type GaN surfaces. To limit the study to the effect of carbon incorporation, we intentionally incorporated carbon into $n$-GaN by using high-temperature annealing of a $\mathrm{SiN}_{x} / \mathrm{CN}_{x} / \mathrm{GaN}$ structure. A carbon-rich $\mathrm{CN}_{x}$ layer was utilized as the source, while $\mathrm{SiN}_{x}$ acted as a capping layer. By using the $\mathrm{CN}_{x}$ layer, we could artificially reproduce the situation in which carbon impurities are included in the $\mathrm{SiN}_{x}$ protection layer of actual device processes. Carbon incorporation was confirmed by secondary ion mass spectrometry (SIMS). The electrical properties of the carbon-incorporated $\mathrm{GaN}$ surface were studied by measuring temperaturedependent current-voltage $(I-V)$ characteristics. As a result, the incorporated carbon behaves as a shallow donor with a binding energy of $30 \pm 20 \mathrm{meV}$ in $\mathrm{GaN}$, which indicates the incorporated carbon mainly substitutes for the Ga. The observed lack of dependence on temperature of the $I-V$ characteristics can be explained with the thin surface barrier (TSB) model.

\section{EXPERIMENTAL}

Figure 1(a) shows the flowchart of the carbon incorporation process in GaN. We used $n$-type $\mathrm{GaN}$ with $\mathrm{Si}$ doping of $1 \times 10^{17} \mathrm{~cm}^{-3}$ grown on a sapphire substrate by metalorganic chemical vapor deposition. The GaN surface was cleaned in the electron-cyclotron-resonance (ECR)-excited $\mathrm{N}_{2}$ plasma. We fabricated the $\mathrm{SiN}_{x} / \mathrm{CN}_{x} / \mathrm{GaN}$ structure by ECR-CVD. A thin $\mathrm{CN}_{x}$ layer with a thickness of 2-10 nm 


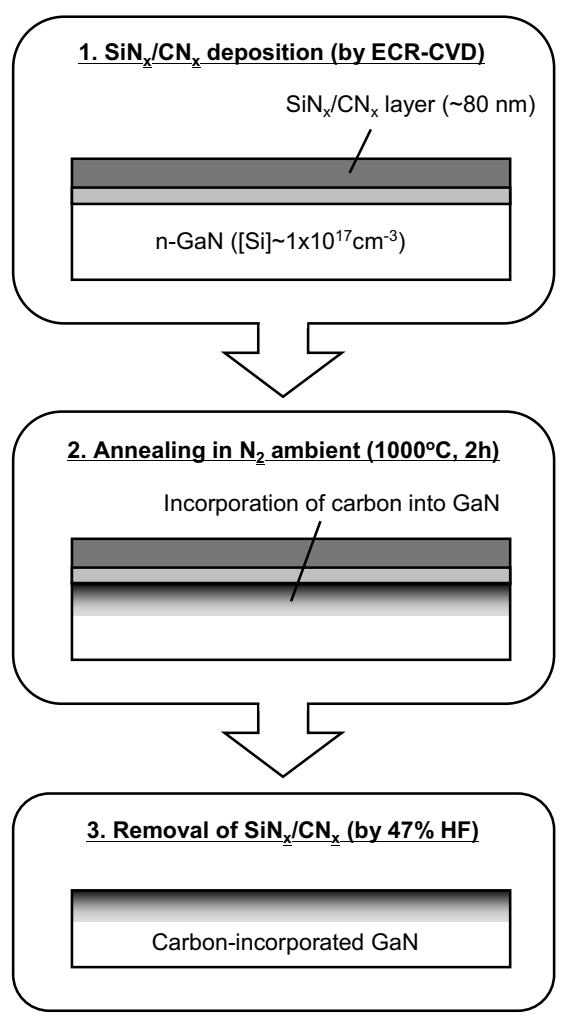

(a)

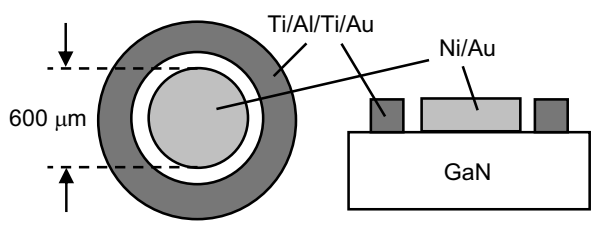

(b)

FIG. 1. (a) Flowchart of carbon incorporation process in GaN. (b) Sample structure of Schottky diode.

was deposited on the $\mathrm{GaN}$ surface at room temperature using a gas mixture of $\mathrm{CH}_{4} / \mathrm{N}_{2}$ as a source. In the subsequent in situ stage, the $\mathrm{SiN}_{x}$ layer was deposited at $260{ }^{\circ} \mathrm{C}$ using a gas mixture of $\mathrm{SiH}_{4} / \mathrm{N}_{2}$. Ellipsometry analysis showed that the thickness and the refractive index of $\operatorname{SiN}_{x}$ were $80 \mathrm{~nm}$ and 2.04, respectively. The sample was annealed at $1000{ }^{\circ} \mathrm{C}$ for $120 \mathrm{~min}$ in a $\mathrm{N}_{2}$ ambient to incorporate the carbon impurity into the $\mathrm{GaN}$ layer. The $\mathrm{SiN}_{x} / \mathrm{CN}_{x}$ bilayer was then removed from the $n-\mathrm{GaN}$ surface in a $47 \%$ HF solution. After the carbon incorporation process, we fabricated circular $\mathrm{Ni} / \mathrm{Au}$ Schottky diodes with $\mathrm{Ti} / \mathrm{Al} / \mathrm{Ti} / \mathrm{Au} \mathrm{Ohmic}$ ring electrodes, as shown in Fig. 1(b). Ni/Au Schottky contacts with a diameter of $600 \mu \mathrm{m}$ were formed by electron-beam deposition. To remove the residual carbon on the GaN surface, we cleaned the GaN surface by using ECR-excited $\mathrm{N}_{2}$ plasma before evaporating the metal contacts.

The chemical properties of the $\mathrm{SiN}_{x}$ and GaN surfaces were characterized using an x-ray photoelectron spectroscopy (XPS) system (ParkinElmer PHI 1600C) with a spherical capacitor and a monochromatic $\mathrm{Al} K \alpha$ radiation source $(h \nu=1486.6 \mathrm{eV})$. The energy scale was calibrated to the Au4f spectrum $(h \nu=84.0 \mathrm{eV})$. The carbon density of the

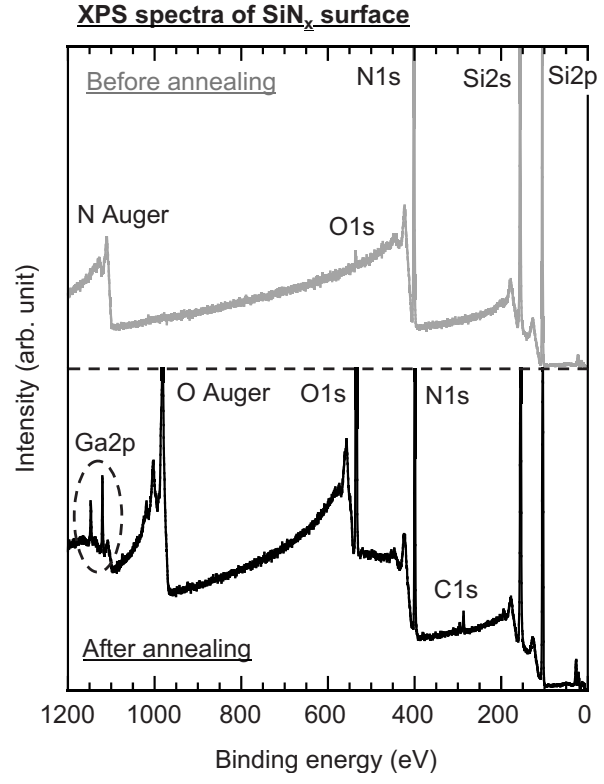

FIG. 2. Comparison of wide-scan XPS spectra of $\operatorname{SiN}_{x}$ surface before and after annealing.

$\mathrm{GaN}$ layer after the incorporation process was characterized using a SIMS system (PHI-6650, ULVAC-PHI Inc.). The temperature-dependent $I-V$ characteristics of the Schottky diodes were measured in a BCT-21MDC semiconductor parameter low-temperature prober system (Nagase \& Co., Ltd.) using an Agilent 4156C semiconductor parameter analyzer. For the carrier concentration measurement, we deposited a $\mathrm{Ti} / \mathrm{Al} / \mathrm{Ti} / \mathrm{Au}$ multilayer on the corner of a square sample and annealed the sample in a $\mathrm{N}_{2}$ ambient at $800{ }^{\circ} \mathrm{C}$ for $1 \mathrm{~min}$.

\section{RESULTS AND DISCUSSION}

\section{A. Ga outdiffusion and carbon incorporation during high-temperature annealing}

Figure 2 compares the wide-scan XPS spectra of the $\mathrm{SiN}_{x}$ surface before and after high-temperature annealing. After annealing, the peaks corresponding to the Ga $2 p$ level appeared in the XPS spectrum. This indicates the outdiffusion of $\mathrm{Ga}$ atoms to the $\mathrm{SiN}_{x}$ layer during the $1000{ }^{\circ} \mathrm{C}$ annealing. Additionally, the full width at half maximum of the Ga $3 d$ XPS spectrum after removing the $\mathrm{SiN}_{x} / \mathrm{CN}_{x}$ bilayer from the annealed $\mathrm{SiN}_{x} / \mathrm{CN}_{x} / \mathrm{GaN}$ structure became wider than the one from the virgin $\mathrm{GaN}$ surface, as shown in Fig. 3. This is also attributed to the decomposition of the GaN surface during the high-temperature annealing. Ga vacancies are known to have relatively low formation energies in $n$-type $\mathrm{GaN} ;{ }^{10,11}$ thus, these characteristics indicate that $\mathrm{Ga}$ vacancies were generated by the high-temperature annealing.

The density profile of incorporated carbon in GaN was investigated using SIMS. Figure 4 shows SIMS profiles of carbon density for virgin and carbon-incorporated samples. The detection limit was determined by measuring the carbon density in an intentionally carbon-implanted GaN sample. The results in Fig. 4 clearly show the incorporation of carbon to a depth of 200-300 $\mathrm{nm}$ for the intentionally incorporated GaN sample. 


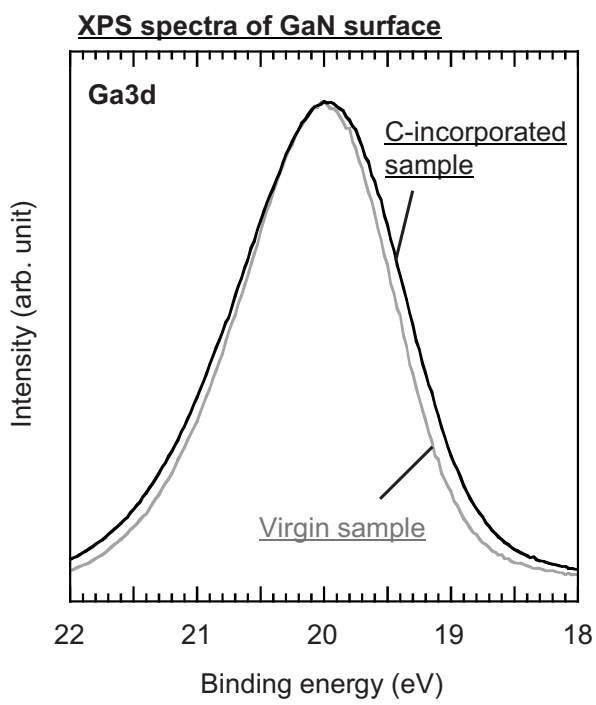

FIG. 3. Comparison of Ga $3 d$ XPS spectra between virgin and carbonincorporated $\mathrm{GaN}$ surfaces.

\section{B. Temperature-dependent $I-V$ characteristics of virgin and carbon-incorporated GaN Schottky diode}

Figure 5 shows the temperature-dependent $I-V$ characteristics of the virgin and carbon-incorporated samples. The carbon-incorporated sample showed a drastic increase in current in both forward and reverse directions. To confirm that this current increase was induced by the incorporated carbon, we compared the $I-V$ characteristics of the carbonincorporated sample with two other samples: one to confirm the influence of high-temperature annealing and the other to confirm the influence of the high-power plasma process in the deposition of the $\mathrm{CN}_{x}$ layer. We fabricated the $\mathrm{SiN}_{x} / \mathrm{GaN}$ and $\mathrm{CN}_{x} / \mathrm{GaN}$ structures by ECR-CVD. The $\mathrm{SiN}_{x} / \mathrm{GaN}$ sample was annealed at $1000{ }^{\circ} \mathrm{C}$ for $120 \mathrm{~min}$ in a $\mathrm{N}_{2}$ ambient; then, the $\mathrm{SiN}_{x}$ layer was removed from the GaN surface in a $47 \% \mathrm{HF}$ solution. The $\mathrm{CN}_{x}$ layer of the $\mathrm{CN}_{x} / \mathrm{GaN}$ sample was removed without high-temperature annealing. The $I-V$ characteristics of the Schottky diodes fabricated on

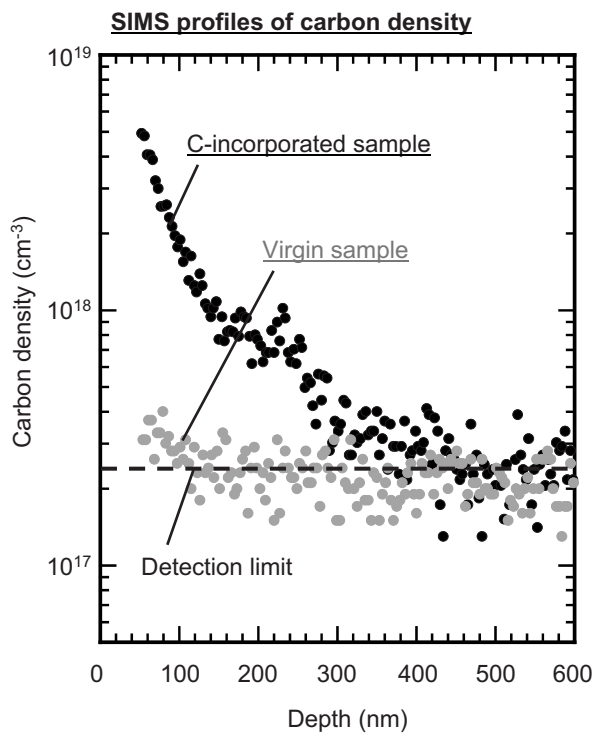

FIG. 4. SIMS profiles of carbon density for virgin and carbon-incorporated samples.

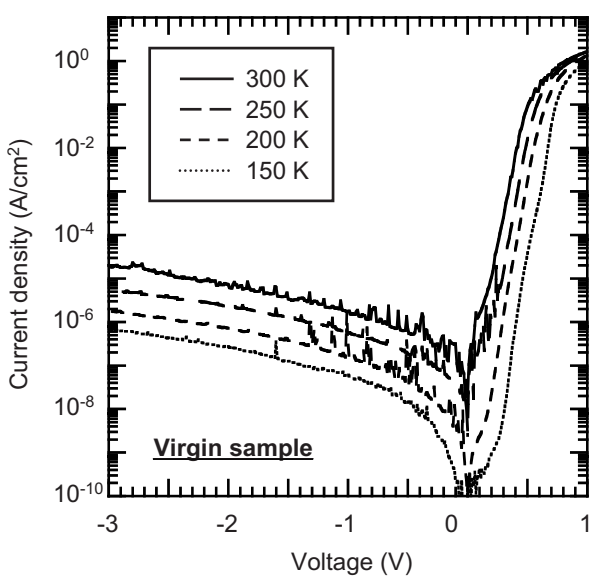

(a)

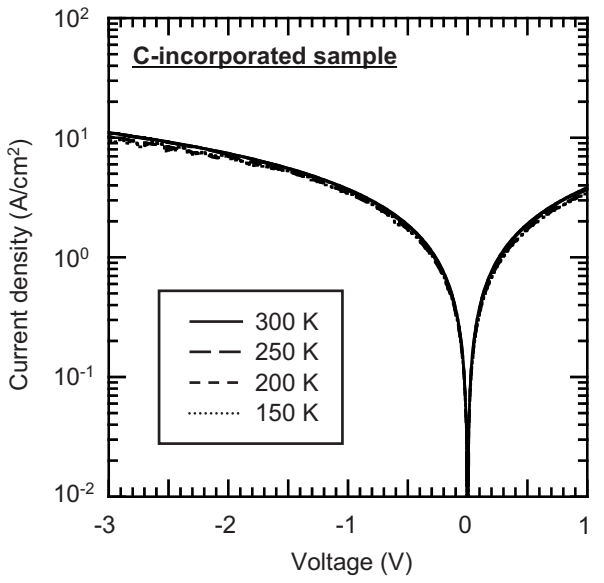

(b)

FIG. 5. Temperature-dependent $I-V$ characteristics of (a) virgin sample and (b) carbon-incorporated sample.

both samples were similar to those of the virgin sample. Hence, the large increase in current of the Schottky diode was induced by incorporated carbon.

In addition, while the $I-V$ characteristics of the virgin sample changed systematically with temperature, the $I-V$ characteristics of the carbon-incorporated sample showed no temperature dependence from 150 to $300 \mathrm{~K}$. This indicates thermionic emission (TE) is the dominant current transport process in the virgin sample. In the carbon-incorporated sample, on the other hand, thermionic field emission (TFE) or field emission (FE) is the major current leakage path in the forward and reverse directions.

\section{Analysis of carbon-incorporated sample based on TSB model}

Figure 6 schematically shows the energy band diagrams under the reverse bias condition and the carrier profiles. Figure 6(a) shows diagrams for the ideal metal $/ n$-GaN Schottky junction, whereas Fig. 6(b) shows the case with surface donors. Hasegawa and co-workers ${ }^{12,13}$ proposed the TSB model for forward and reverse current transport in GaN Schottky barriers. This model assumes that there are high-density donors near the Schottky interface; this reduces the width of the Schottky potential, thereby increasing the number of electrons tunneling through the thin surface barrier in both direc- 

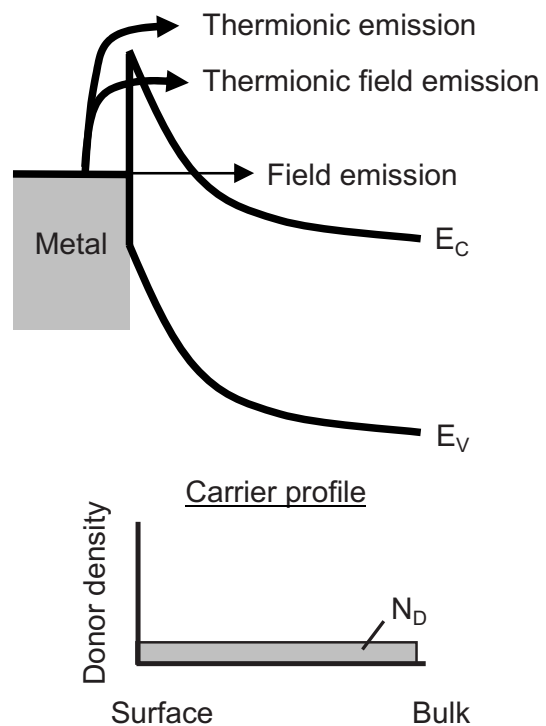

(a)
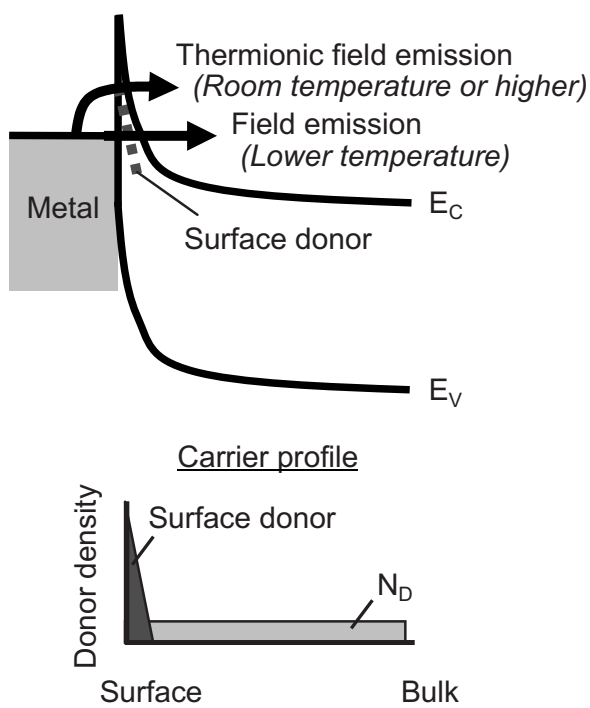

(b)

FIG. 6. Schematic illustrations of energy band diagram under the reverse bias condition and carrier profile for (a) the ideal metal $/ n-G a N$ Schottky junction and (b) the case with surface donors.

tions. Therefore, either the TFE or FE mechanism becomes dominant and reduces the temperature dependence. It is likely that the substitutional carbon at the Ga site behaves as a shallow donor with a binding energy of $30 \mathrm{meV}$ in $\mathrm{GaN},{ }^{5,14}$ and high-density substitutional $\mathrm{C}_{\mathrm{Ga}}$ exists in the surface region; thus both forward and reverse currents in the carbonincorporated sample would increase. If the incorporated carbon substituted for $\mathrm{N}$, the substitutional $\mathrm{C}_{\mathrm{N}}$ would behave as an acceptor and compensate for the donor. However, the experimental data showed no such phenomenon. One of the reasons is that while Ga vacancies are more favorable under $n$-type conditions, the formation energy of the $\mathrm{N}$ vacancy is large in $n$-type conditions. ${ }^{10,11}$ Thus, there is a possibility that few $\mathrm{N}$ vacancies are generated during high-temperature annealing and that $\mathrm{Ga}$ vacancies are generated instead.

We tried to reproduce the experimental data by calculating the $I-V$ characteristics based on the TSB model analysis

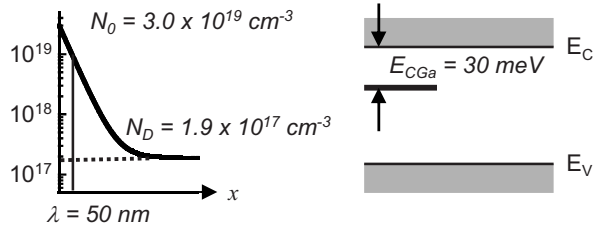

(a)

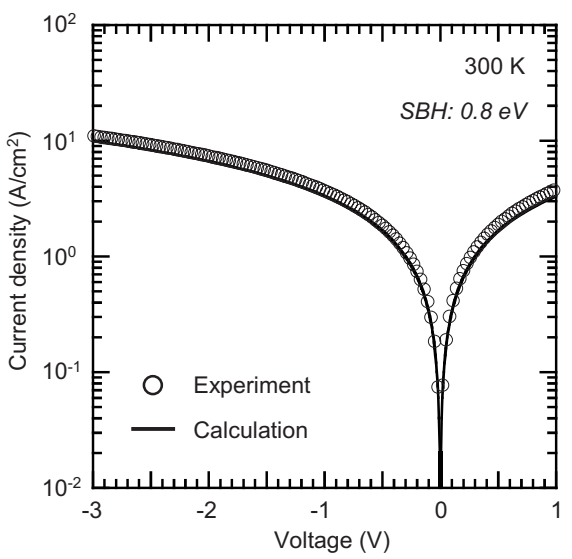

(b)

FIG. 7. (a) Best choice of distribution shape and parameter values to reproduce the experimental data of the carbon-incorporated sample. (b) Calculated $I-V$ curves (solid curves) fitted to experimental data (symbols) of the carbon-incorporated sample.

of Kotani et al. ${ }^{13}$ The substitutional $\mathrm{C}_{\mathrm{Ga}}$ near the surface was assumed to have the following exponential distribution:

$$
N_{\mathrm{CGa}}(x)=N_{0} \exp \left(-\frac{x}{\lambda}\right)
$$

where $x$ is depth from surface, $N_{0}$ is the peak density of $\mathrm{C}_{\mathrm{Ga}}$ at the surface, and $\lambda$ is decay constant. Another important parameter, the energy depth, $E_{\mathrm{CGa}}$, of the substitutional $\mathrm{C}_{\mathrm{Ga}}$ was varied as a fitting parameter. The bulk shallow donor density $N_{\mathrm{D}}$ was set to be that of the experimental sample of $N_{\mathrm{D}}=1.9 \times 10^{17} \mathrm{~cm}^{-3}$. Hirota et al. ${ }^{5}$ estimated the binding energy of the $\mathrm{C}$ donor level to be $30 \mathrm{meV}$ (the substitutional $\mathrm{C}_{\mathrm{Ga}}$ ). Wang and Chen ${ }^{14}$ also predicted the ionization energy of the $\mathrm{C}_{\mathrm{Ga}}$ donor to be $34.0 \mathrm{meV}$ from the calculation using the effective-mass approximation. In reference to these results, we used the value of $30 \mathrm{meV}$ for the $\mathrm{C}$ donor level in our calculation to reproduce the experimental $I-V$ data. Then, as shown in Fig. 7, we obtained the excellent fitting result for the carbon-incorporated GaN Schottky diode by assuming the exponentially decaying distribution shown in Fig. 7(a). The simulation almost completely reproduced the forward and reverse $I-V$ behavior. In addition, the $\mathrm{C}_{\mathrm{Ga}}$ distribution is similar to the carbon density profile obtained by SIMS in Fig. 4. From the separate calculation by changing the value of the energy level, we found that the accuracy of the energy level was $30 \pm 20 \mathrm{meV}$. The calculated $I-V$ characteristics for the virgin sample also reproduced the experimental data by using the same parameters of Schottky barrier height $(\mathrm{SBH})=0.80 \mathrm{eV}$ and $N_{\mathrm{D}}=1.9 \times 10^{17} \mathrm{~cm}^{-3}$ as the carbonincorporated sample. 


\section{Two-layer Hall analysis of carbon-incorporated sample}

Finally, we measured the change in carrier concentration of the GaN layer before and after the carbon incorporation process. The carrier concentration of the virgin $n-\mathrm{GaN}$ was $1.9 \times 10^{17} \mathrm{~cm}^{-3}$. After incorporation, it increased to 3.0 $\times 10^{17} \mathrm{~cm}^{-3}$. However, this result is for the whole sample with a thickness of $3 \mu \mathrm{m}$ consisting of an upper carbonincorporated layer and a GaN under layer. Thus, we calculated the carrier concentration of only the upper carbonincorporated layer by using two-layer Hall analysis. ${ }^{15-17}$ The carrier concentration of upper carbon-incorporated layer, $n_{\mathrm{ci}}$, is given as follows:

$$
n_{\mathrm{ci}}=\frac{1}{d_{\mathrm{ci}}} \frac{\left(n \mu d-n_{\mathrm{GaN}} \mu_{\mathrm{GaN}} d_{\mathrm{GaN}} t\right)^{2}}{n \mu^{2} d-n_{\mathrm{GaN}} \mu_{\mathrm{GaN}}^{2} d_{\mathrm{GaN}} t} .
$$

Here, $n$ and $n_{\mathrm{GaN}}$ are the carrier concentrations of the whole sample and $\mathrm{GaN}$ under layer, $\mu$ and $\mu_{\mathrm{GaN}}$ are the carrier mobilities of the whole sample and GaN under layer, $d, d_{\mathrm{ci}}$, and $d_{\mathrm{GaN}}$ are the thicknesses of the whole sample, upper carbon-incorporated layer, and GaN under layer, and $t$ is the correction factor related to the series resistance between the upper and under layers. The parameters of the $\mathrm{GaN}$ under layer were those of the virgin $\mathrm{GaN}$ results.

When the thickness of carbon-incorporated layer was taken from the SIMS profile to be $200 \mathrm{~nm}$, the carrier concentration of the carbon-incorporated layer was calculated to be $2.4 \times 10^{18} \mathrm{~cm}^{-3}$. Even in this case, the carrier concentration near the surface $(\sim 50 \mathrm{~nm})$, which has more of an effect on the Schottky contact, was estimated to be 1.0 $\times 10^{19} \mathrm{~cm}^{-3}$. This value is consistent with the SIMS results (Fig. 4) and the assumed carrier profile [Fig. 7(a)]. Such high-density shallow donors (incorporated carbon) will reduce the width of the Schottky potential and increase the tunneling currents through the Schottky barrier. In relation to the increase in carrier concentration near the surface after the carbon incorporation, we observed a slight decrease in the sheet resistivity for the present sample. When we used an undoped $n$-type GaN layer with a different thickness, the sheet resistivity was found to increase slightly. ${ }^{18}$ This discrepancy may arise from the difference of crystalline quality among the GaN layers. In the undoped GaN layer, it is likely that the $n$-type conduction originates from the existence of nitrogen vacancies or oxygen impurities. In this case, there is a possibility that the incorporated carbon can form deep levels in complex configuration with such residual defects or impurities. This may result in a partial compensation of the residual donors in undoped $\mathrm{GaN}$, leading to the slight increase in resistivity.

\section{CONCLUSIONS}

We intentionally incorporated carbon into $n$-GaN by using high-temperature annealing of a $\mathrm{SiN}_{x} / \mathrm{CN}_{x} / \mathrm{GaN}$ structure to study the effect of unintentional incorporation of carbon on the electrical properties of $n$-type GaN. The main conclusions are as follows.
(1) The XPS results showed out diffusion of Ga atoms from the GaN surface during high-temperature annealing even when the $\mathrm{SiN}_{x}$ layer existed.

(2) The SIMS results clearly showed incorporation of carbon into GaN to a depth of 200-300 nm.

(3) The $I-V$ characteristics showed a drastic increase in current in the forward and reverse directions of the Schottky diode in the carbon-incorporated sample. They also showed no temperature dependence from 150 to $300 \mathrm{~K}$.

(4) The measured $I-V$ curves of the carbon-incorporated sample could be almost completely reproduced in the forward and reverse directions by assuming an exponentially decaying distribution from the surface for the shallow donors with an energy depth of $30 \mathrm{meV}$.

(5) The carbon incorporated during the high-temperature annealing mainly substituted for $\mathrm{Ga}$, and this substitutional $\mathrm{C}_{\mathrm{Ga}}$ behaved as a shallow donor in $\mathrm{GaN}$, leading to a high-density doping effect near the GaN surface. This reduced the width of the Schottky potential, allowing electrons to tunnel thorough the thin barrier.

In conclusion, unintentional incorporation of carbon impurities during GaN device fabrication process may induce current leaks in Schottky contacts. Therefore, the device should be carefully made so that the actual device process does not unintentionally incorporate carbon.

\section{ACKNOWLEDGMENTS}

One of the authors (T.K.) would like to thank the Japan Society for the Promotion of Science (JSPS) Research Fellowships for Young Scientists.

${ }^{1}$ H. T. Wang, L. S. Tan, and E. F. Chor, Thin Solid Films 515, 4476 (2007).

${ }^{2}$ K. Nomoto, T. Tajima, T. Mishima, M. Satoh, and T. Nakamura, IEEE Electron Device Lett. 28, 939 (2007).

${ }^{3}$ D. Ozaki, J. Ebihara, Y. Ohshima, R. Takeuchi, and T. Inada, Nucl. Instrum. Methods Phys. Res. B 257, 320 (2007).

${ }^{4}$ H. Ito and T. Ishibashi, Jpn. J. Appl. Phys., Part 2 30, L944 (1991).

${ }^{5}$ R. Hirota, K. Kushida, J. Takahashi, and K. Kuriyama, Nucl. Instrum. Methods Phys. Res. B 219, 792 (2004).

${ }^{6}$ A. Armstrong, C. Poblenz, D. S. Green, U. K. Mishra, J. S. Speck, and S. A. Ringel, Appl. Phys. Lett. 88, 082114 (2006).

${ }^{7}$ D. J. As, U. Kohler, M. Lubbers, J. Mimkes, and K. Lischka, Phys. Status Solidi A 188, 699 (2001).

${ }^{8}$ C. H. Seager, A. F. Wright, J. Yu, and W. Gotz, J. Appl. Phys. 92, 6553 (2002).

${ }^{9}$ T. Hikosaka, T. Narita, Y. Honda, M. Yamaguchi, and N. Sawaki, Appl. Phys. Lett. 84, 4717 (2004).

${ }^{10}$ S. Limpijumnong and C. G. Van de Walle, Phys. Rev. B 69, 035207 (2004).

${ }^{11}$ M. A. Reshchikov and H. Morkoc, J. Appl. Phys. 97, 061301 (2005).

${ }^{12}$ H. Hasegawa and S. Oyama, J. Vac. Sci. Technol. B 20, 1647 (2002).

${ }^{13}$ J. Kotani, T. Hashizume, and H. Hasegawa, J. Vac. Sci. Technol. B 22, 2179 (2004).

${ }^{14}$ H. Wang and A.-B. Chen, J. Appl. Phys. 87, 7859 (2000).

${ }^{15}$ R. L. Petritz, Phys. Rev. 110, 1254 (1958).

${ }^{16}$ D. C. Look, C. E. Stutz, and C. A. Bozada, J. Appl. Phys. 74, 311 (1993).

${ }^{17}$ B. Arnaudov, T. Paskova, S. Evtimova, E. Valcheva, M. Heuken, and B. Monemar, Phys. Rev. B 67, 045314 (2003).

${ }^{18}$ T. Kimura, S. Ootomo, T. Nomura, S. Yoshida, and T. Hashizume, Jpn. J. Appl. Phys., Part 2 46, L224 (2007). 